\title{
Programming to Promote Information Literacy in the Era of Fake News
}

\author{
Caroline L. Osborne ${ }^{1}$
}

\begin{abstract}
Given the instant availability of information and the proliferation of questionable news, the ability to critically examine information before consuming it, is of increasing importance. The need for excellent information literacy skills is evident but lacking. This article highlights reasons as to resistance to existing information literacy efforts and suggests components for information literacy programming with a focus on fake news.
\end{abstract}

\section{INTRODUCTION}

The concept of veracity in the world of online information is not novel to information professionals. Debate, dialogue, and programming around the quality of information dates back to the 1960s. The earliest discussions on the topic of literacy, and the coining of the term information literacy, evolve out of the reform movements begun in the 1960 s emphasizing critical thinking and learning to learn. ${ }^{2}$ One of the initial uses of the term information literacy is credited to a 1974 paper by Paul Zurkowski, former president of the U.S. Information Industry Association. ${ }^{3}$ Concerns regarding fake news abounded during and following the 2016 United States Presidential election.

The traditional definition of fake news is associated with yellow journalism and implies fabricated news. There is a specific element of deception or intent to deceive implicit in this definition. However, this definition also predates our most recent election. When fake news and information literacy are combined, they raise similar questions of information literacy and media literacy. Media literacy is a subtenant of information literacy specifically focusing on possessing the knowledge needed to facilitate access to media (both old, as in print, and new, as in digital), understanding of the media, and the ability to create and/or express oneself using media. ${ }^{4}$ Information literacy, fake news, and media literacy all ask the same key questions: What is an information literate consumer, and how to we educate to create an information literate population that may thrive in today's media intensive culture?

The initial step is to define information and media-literate consumers as those who possess the skills necessary to successfully recognize and use information and media. The United Kingdom defines an information-literate

\footnotetext{
${ }^{1}$ Caroline L. Osborne, Esq., Assistant Dean of Legal Information Services and Professor of Legal Research, Washington and Lee University School of Law. This symposium article documents the presentation given at the 2017 IALL Conference at Emory University in October 2017. It represents part three of a panel presentation on the topic of Fake News and Information Literacy with a focus on educational programming on information literacy with a concentration on fake news.

${ }^{2}$ Forest Woody Horton, JR., United Nations Educational. Scientific and Cultural Organization, Understanding INFORMATION LitERACY: A PRIMER (2007).

${ }^{3} I d$.

${ }^{4}$ Media literacy facilitates "having a critical relationship to media content in a time when the media constitutes one of the most powerful forces in society." $I d$. Media literate persons will possess the ability to contribute to and participate in society be active citizens. $I d$.
} 
consumer as one with the ability to choose and use communication products effectively. ${ }^{5}$ The Medical Library Association defines "health information literacy" as the set of abilities needed to recognize a health information need, identify likely information sources and use them to retrieve relevant information, assess the quality of information and its applicability to a specific situation, and analyze, understand, and use information to make good health decisions. ${ }^{6}$ One suggestion for a working definition of an information- and media-literate consumer is one who possesses the skills to find and evaluate information as fit for a specific need, distinguishes reliable from unreliable sources, and recognizes satire, hoaxes, and propaganda as differing forms of information fit for specific purposes and uses.

Building on the definition of an information literate consumer, this article addresses two specific points in broader discussion of the education of consumers to be information literate in the era of fake news. The first point addresses the seeming disconnects with existing education efforts, with a focus on the existing impasse between current educational efforts and the creation of information-literate consumers. The second point addresses information literacy programming in 2018 with a specific look at the elements an effective information literacy program with a concentration on news and media literacy should include.

\section{The Educational Impasse and an Information-Literate Public}

Dialogue concerning the ability to find and effectively use information and related programing is neither novel nor rare. The academic community has long recognized the power of knowledge and the need to equip the population of the information age to effectively employ information. ${ }^{7}$ The elephant in the room is why information literacy is lacking in spite of existing educational efforts? Five logical points of impasse stand between information literacy and an information-literate public. They are:

1. An attention deficit regarding need to be information literate (the "Attention Deficit").

2. An awareness deficit of the need to examine and read critically the information one consumes.

3. The existence of a confirmation bias in regards to information consumed.

4. The increasing sophistication of the business model for fake news or inferior information.

5. A fundamental change in how we acquire information.

If the goal is to create an information-literate public that possesses the skill to evaluate information for authenticity and fit prior to use, then one must address the points of impasse.

\section{A. Attention Deficit}

In 2000, the Association of College and Research Libraries (ACRL) defined information literacy as "a set of abilities requiring individuals to recognize when information is needed and have the ability to locate, evaluate, and use effectively the needed information." 8 Despite the recognized importance of the skill, satisfaction with the mastery of the skill continues to be elusive. This prompts the question of how does one attract the attention of those who need information literacy education the most? Raising awareness to the need is critical to breaching the impasse. Consider a United Kingdom statistic that reveals 3.2 million adults fall victim to deceptive, unsolicited mailings, phone calls, or email. ${ }^{9}$ The overwhelming suggestion is that an information literate public is lacking despite ongoing librarian and other educational efforts. ${ }^{10}$ This conclusion leads to the question of why education efforts of the past failed to create an information literate public? An absence of attention by the consumer to the quality of the information consumed and a need to confirm the veracity of information are pieces to the answer to this question.

${ }^{5}$ Colette Bowe, What is Consumer Literacy and Why Does It Matter? (Feb. 2007) http://www.ofcomsunsumerpanel.org.uk.

6 Jean P. Shipman, Sabrina Kurtz-Rossi \& Carla J. Funk, The Health Information Literacy Research Project, 97 J. Med. LiBr. Assoc. 293, 293-94 (2009).

${ }^{7}$ Michelle Hale Williams \& Jocelyn Jones Evans, Factors in Information Literacy Education, 4 J. OF PoL. ScI. Educ. 116, 116 (2008).

${ }^{8} I d$.

9 Bowe, supra note 5.

${ }^{10} \mathrm{Id}$. 


\section{B. Awareness Deficit in the Critical Consumption of Information}

Once there is attention to the need to be information literate, the next impasse is acceptance of the need to examine and read critically information prior to use. The Robert R. McCormick Foundation at Stanford studied young persons' ability to reason about information derived from the internet and described it as "bleak." 11 One particular finding stated that when evaluating information that flows through social media, youth are easily duped. ${ }^{12}$ Where once people relied on publishers, editors, and subject-matter experts to vet information, in the era of the unregulated internet all bets are off. ${ }^{13}$ Knowing that one must pay attention to the quality of information is but one part of raising awareness. All too often we grab information because it is convenient or we believe it comes from a trusted source. Raising the awareness of the need to examine information, even information that we believe is of a good quality, is key. Habituation of the consumer to examine information critically prior to acceptance is the goal.

\section{Confirmation Bias and the Filter Bubble}

People like stories that reinforce their world-view, making them more likely to accept or buy into information that fits their preconceived views. ${ }^{14}$ Confirmation bias makes one less likely to challenge the information found and more accepting of information as it fits. ${ }^{15} \mathrm{We}$ may be aware that we need to verify information and understand that we need to pay attention to the source and quality of the information we consume, but humans are, well, human, and like to accept information that fits with their conception of the world. More troubling is, in an era where the trust filters of editors and publishers are vanishing, the information consumer increasingly relies on friends and family. This factor exacerbates the confirmation bias. If Mom, Dad, or best friend tells us it is so, then it must be so.

The filter bubble compounds the impact of confirmation bias. The filter bubble is a phenomenon of the personalized search engine functions of search engines like Google and products such as Facebook. ${ }^{16}$ It is an artifact of technology and the algorithms a web site employs to push information to the visitor. ${ }^{17}$ The goal of the algorithm is to select and push information to the visitor that it believes the visitor will favor based on prior behavior. The impact of this is intellectual isolation due to an echo chamber that feeds the visitor a diet of information screened to eliminate competing voices. ${ }^{18}$

When confirmation bias and the impact of the filter bubble combine, the result is easy and natural acceptance of information that fits our view of the world and is whitewashed for dissent. The corollary is that one adopts new trust indicators and must be pushed to critically examine that which one is naturally inclined to accept. This presents a significant challenge to overcome when one encounters a falsehood, especially a well-constructed falsehood.

\section{Sophisticated Business Model}

The business model surrounding the fake news industry is increasingly sophisticated resulting in a need for greater skills in detecting poor information. The business of fake news is lucrative, directly linking revenue with advertising and clicks and creating revenue of as much as $\$ 30,000$ per month. ${ }^{19}$ As a key motivation is to make

${ }^{11}$ Sam Weinberg et al., Stanford History Education Group, Evaluating Information: The Cornerstone of Civic Online Reasoning: Executive Summary (Nov. 22, 2016) https://www.purl.stanford.edu/fv751yt5934.

${ }^{12} I d$.

${ }^{13} \mathrm{Id}$.

${ }^{14}$ Interview by Christina Pazzanese with Cass Sunstein, Harvard Law School, MA (Mar. 24, 2017).

${ }^{15} I d$.

${ }^{16}$ Kalev Leetaru, Why 2017 Was the Year of the Filter Bubble? ForBes (Dec. 18, 2017) https://www.forbes.com/sites/kalevleetaru/2017/2018/why-was-2017-the-year-of-the-filter-bubble/26146572746b.

${ }^{17}$ Filter Bubble, TeChOPEDIA.com (March 21, 2018 at 3:57 PM) http://techopedia.com.

${ }^{18} I d$.

${ }^{19}$ Laura Sydell, We Tracked Down a Fake-News Creator in the Suburbs. Here's What We Learned, NPR All Things Considered (Nov. 23, 2016) http://www.npr.org/sections/alltechconsidered/2016/1/23/503146770/npr-rinds-the-head-of-acovret-fake-news-operation-in-the-suburbs). See also, Andrew Higgins, Mike McIntire \& Gabriel J.S. Dance, Inside a Fake News Sausage Factory: 'This is All About Income', N.Y. Times (Nov. 25, 2016) http://nytimes/2go46P0. 
money and attract viewers to the site, this, in turn, leads to sophisticated webpages designed to be believable to the audience and entice one to click. The more believable the page the more likely the consumer is to accept what he or she finds as good information with minimal resistance or critical analysis. The end result is a sea of information that appears to be truthful but is deceptive. Excellent skills are required to protect the consumer of the information from the deception.

\section{E. The Impact of the Web and Social Media on the Acquisition of Information}

The impact of the web and social media on how and where we consume information is another impasse to identifying false information. According to a 2016 Pew Research Center study, $62 \%$ of adults obtain their news from a social media platform. ${ }^{20}$ By comparison, that same number was $49 \%$ in $2014 .{ }^{21}$ Instead of the local or national paper or the nightly news broadcast from ABC, NBC, or CBS, $66 \%$ get their news from Facebook, $59 \%$ from Twitter, and $70 \%$ from Reddit. ${ }^{22}$ Gone is the intermediary responsible for fact checking. When confirmation bias is added to the equation, the concern becomes even greater. Who is checking the veracity of the information?

Consider the design of a smart phone. Manufacturers such as Apple design their devices to grab your attention. ${ }^{23}$ One statistic from Apple suggests that users look at their phone eighty times a day. ${ }^{24}$ The device is useful and has an unprecedented hold on our attention.

Perhaps of even greater concern, is how the allure of the device impacts the manner in which our brain processes information. In a UC San Diego study, the ability to interpret and solve an unfamiliar problem was tested. ${ }^{25}$ Those with proximity to a device performed the worst. ${ }^{26}$ The conclusion of the study was that integration of the phone into daily life was a brain drain that could diminish learning, logical reasoning, abstract thought, problem solving, and creativity. ${ }^{27}$ Our brains are dependent upon technology. Instead of remembering a phone number we reach for the phone. In lieu of maps for directions we rely on GPS technology. There is little need to commit information to memory when the device with Google is at hand. We are leaving remembering to technology, which impacts our thinking. Our reliance on the device is diminishing the rich intellectual associations that form the essence of personal knowledge and give rise to critical and conceptual thinking. ${ }^{28}$ This has the significant and long-term impact of diminishing our critical reasoning skills. ${ }^{29}$ When we constrict our capacity for reasoning and recall or transfer those skills to a gadget, we sacrifice our ability to turn information into knowledge. We get the data but lose the meaning.

Also, we are creating a gullibility crisis. The gullibility crisis is acerbated by our habit of acquiring our news and information directly from the internet in the absence of any filters for accuracy. When one combines the fact that the business model for fake news is increasingly sophisticated and lucrative and the public increasing lacks the skill to distinguish good information from bad, the result is an environment in which people are all too quick to accept lies and half-truths consumed from social media. Credit for this goes, in part, to the device, which has sapped the power of discernment. People will believe anything the phone tells them.

\section{Designing an Effective Information Literacy Program with a News Focus}

\section{A. The Components}

Acknowledging the impasse to educational efforts, the looming question is how to design an effective literacy program. Basic elements of any effective program include: source evaluation, recognition of opinion and

\footnotetext{
${ }^{20}$ Jeffrey Gottfried \& Elisa Shearer, Pew Research Center, News Use Across Social Media Platforms 2016 (2016).

${ }^{21} I d$. (the 2014 question was worded in a slightly different manner to focus on seeing versus getting).

22 Id.

${ }^{23}$ Nicholas Carr, How Smartphones Hijack our Minds, Wall ST. J. (Oct. 10, 2017).

${ }^{24} I d$.

${ }^{25} I d$.

${ }^{26} I d$.

${ }^{27} \mathrm{Id}$.

${ }^{28} \mathrm{Id}$.

${ }^{29} I d$.
} 
bias, veracity of the claims, and a discussion of tools to assist in determining veracity, currency, and expertise and identity of the author. These are the common themes of any information literacy program. Designing a program to provide information consumers with the skills to critique news requires additional components focused on news. A program focused on finding, critiquing, and using news stories must include the additional elements of:

1. defining fake news;

2. addressing the role of social media in the consumption of news which includes the spreading of news; and

3. drawing distinctions among, straight news, opinion pieces, news satire, news parody, fabrication of news and manipulation of news and misappropriation.

These elements are must additions, but the program designer may determine that the skill of recognizing propaganda and advertising is also worthy of inclusion.

Defining fake news should be done at the start of any program. As suggested in the Introduction to this article, fake news is not new. Instances of fake news date back to the invention of the printing press, if not before, and are traditionally associated with yellow journalism. ${ }^{30}$ It is important to update this definition for 2018 given the prevalence of the use of the term in today's discourse and the fact that the term is something of a buzzword. A traditional definition of the term might be "news articles that are intentionally and verifiably fake, and could mislead readers." ${ }^{31}$ An updated definition might broaden the definition to address use of the term as a means of discrediting critical reporting of otherwise accurate news stories. ${ }^{32}$ Traditionally, a good-faith error on the part of a journalist is not credited as creating a false narrative or fake news piece, especially when the error is acknowledged and corrected; however, in today's hyper-sensitive news culture, such inaccuracies are often credited as fake news. ${ }^{33}$

Creating a common definition at the start of any program is critical to promote thought and engage the audience in the discussion. In a program where we discuss the concept of confirmation bias and challenge people to critically read stories that complement their own world-view, it is important to create that common ground. Is a story with an innocent error false? Is intent to deceive a required element? Where is the line between the outrageous and deliberately misleading and the innocent? What is outrageous? Discussion around creating a common definition serves to begin to open the minds of the audience and engage them in a meaningful manner. One must distinguish bad news from fake news. Bad news is the result of poorly researched, error filled, and simply shoddy reporting. It is distinct from fake news, as bad news is the result of poor quality and inferior work. Fake news has the specific element of deception or intent to mislead included. Both bad and fake news should be dismissed by the information literate consumer but for different reasons. ${ }^{34}$

Acquisition of information and news, specifically, has fundamentally changed. Where news was once obtained from a local, regional, or national paper that employed trusted journalists and editors or from the nightly network newscast, social media, and Facebook, in particular, are today's frequent sources of news stories. Building an understanding of the need to seek out the original source of the news item to determine its veracity even when the item appears on the Facebook page of a trusted friend, mentor, or family member is key.

This is also an excellent time to discuss the concept of confirmation bias and the filter bubble. We like to read stories that reinforce our view of the world and what is better than when a trusted friend or family member directs you to the news item? Their endorsement serves to make the item trustworthy even if it is not. This is problematic in today's media culture where (1) parody, opinion, and satire are easily and often confused and we have infotainment, (2) the traditional fact checkers of the news editor and publisher are missing from the equation or considered to be disreputable based on the political climate, and (3) we live in a culture of instant gratification and want what we want when we want it. People are acculturated to accept an answer, any answer, and not look for a good answer.

What is satire? What is parody? What does it mean to misappropriate? What is an opinion? What do we mean by manipulation? Education to recognize these in the context of news is the next fundamental element of a program. Have you ever stood around the water cooler and heard a discussion of the prior evening's The Daily

${ }^{30}$ Joanna M. Burkhardt, History of Fake News, 53 LiBr. Tесн. ReP. 5 (2017).

${ }^{31}$ Hunt Allcott \& Matthew Gentzkow, Social Media and Fake News in the 2016 Election, 31 J. of Econ. Persp. 211 (2017).

${ }^{32}$ Edson C. Tandoc Jr., Zheng Wei Lim \& Richard Ling, Defining "Fake News", 6 Digital Journalism 137, 138 (2018).

${ }^{33} \mathrm{Id}$.

${ }^{34}$ Eugene Kiley \& Lori Robertson, How to Spot Fake News, THE WIRE (Nov. 18, 2016) http://www.factcheckorg/2016/11/ how-to-spot-fake-news. 
Show discussed as a factual news event? Discussion of current events is a natural for late-night television and comedy, but drawing the distinction between entertainment and news is critical. Infotainment is not your trusted news source, no matter how much you love the host or how funny the host is. Providing examples of satire, parody, misappropriation, and opinion journalism and distinguishing them in the context of news, entertainment, and fake news builds the set of critical reasoning skills needed to facilitate an information-literate consumer.

If there is additional time allotted in your program, consider a discussion of advertising, propaganda, and public relations. Propaganda and advertisement, clearly, are not news but they regularly employ elements that are easily confused in today's presentation of news. Even excellent news sources publish a mixture of pieces from strong political pieces to silly quizzes. This mixed presentation makes it difficult for the less savvy to gauge the authority of a piece. Given that a news site may include paid advertising that is styled as a story rather than an advertisement the unsophisticated consumer may be easily confused. Information is often opaque and easily conflated with things that masquerade as news. Propaganda by its nature is likely to employ deceptive elements designed to confuse. Find a popular news site and engage the audience with classifying the different information pieces as a means of building the critical reasoning skills needed to distinguish among straight news and advertisement.

\section{B. The Journalists}

Journalism shares the burden and desire of creating an information-literate public and journalists are the news experts. Like information professionals, journalists share a deep commitment to verified sources and reliable information. These similar and shared values for reliable information make journalism an excellent partner in the quest for developing literacy skills. Borrow from journalism those things that the field does well: content or source evaluation, education of perspective and bias, original sourcing and ethics.

Evaluate the substance of the article. Design your program to build the skill of critical reading. Teach consumers to examine the substance of the piece for hallmarks of quality rather than superficial characteristics such as professional appearance of the site or domain name. Persons who rely on the superficial rather than the actual content are more likely to miss a piece of satire or a partisan opinion piece absent a critical read of the content. Borrow from the journalist and outline those elements that are hallmarks of a quality news story. Items such as the sourcing and crediting of the facts in a story, the tone of a story - is the tone neutral or intended to persuade - are all the facts sourced in the story, was the story reported in a timely fashion, is the story balanced and does it present as fair? ${ }^{35}$ Does the story incite your passion? Is it too good or outrageous to be true?

Differentiating between perspective and bias is another tenant we may borrow from journalism. Writing from a point of view is not the same as cherry-picking facts and twisting a story unfairly. ${ }^{36}$ Read with an open mind. Distinguish between editorials, opinions, and commentaries intended to be argumentative and express a strong opinion and news stories that should not. Good journalism does not mix the two, which suggests if the two are mixed, the piece fails to be good journalism.

The profession of journalism requires reporters to be thorough and dig deep to the original source. Again, we should borrow this trait from our colleagues. Programming should stress finding the original source of the story. Discuss how stories are published and syndicated. Examine the practice of harvesting and repackaging of a story without additional research. Emphasize that this practice is not unusual. Caution the consumer to consider the distance between the original reporting with the wariness that the greater the distance the greater the chance for corruption of the original story.

Borrow from the Journalist Code of Ethics. Specifically, did the author verify the facts. Is the story overly simplified or sensationalized? Is the headline even. How are anonymous sources used? The Society of Professional Journalist was originally founded in 1909 for the purpose of improving and protecting journalism. ${ }^{37}$ The SPJ Code of Ethics is a statement of four "abiding principles": (1) seek truth and report it, (2) minimize harm, (3) act independently, and (4) be accountable and transparent. ${ }^{38}$ Each principle is supplemented by additional explanatory

\footnotetext{
${ }^{35}$ Reporting and Writing Basics (March 21, 2018, 2:00 PM) http://www.handbook.reuters.com .

${ }^{36} \mathrm{Id}$.

${ }^{37}$ Society of Professional Journalists, SPJ Code of Ethics (March 20, 2018, 3:20 PM) http://www.spj.org.

${ }^{38}$ Id.
} 
material. ${ }^{39}$ Consider the statements of accepting responsibility for the accuracy of their work, the duty to verify information prior to release, and the duty of care to not misrepresent or oversimplify a story. Also, charge the consumer to identify sources clearly to permit the public to responsibly judge the content of the article. These are all indicia of an ethical story, responsible journalism, and indicia of content with a high degree of trust. If a story is lacking then the absence of such indicia should indicate concern over reliance and use of the content.

Educate the consumer to reverse engineer the story. If the consumer understands how a quality story is put together then they have the skill set to challenge and dissect the suspicious. This critical analysis is what serves to make a consumer information literate.

\section{Association of College and Research Libraries ("ACRL") and American Association of Law Libraries ("AALL")}

Both ACRL and AALL are leaders in the discussion of information literacy. Significantly, together they define information literacy, legal information literacy, and the skill set an information literate person should possess. ACRL denotes six essential skills that define an information-literate person. They are:

1. The ability to determine the extent of the information needed

2. The ability to access needed information effectively and efficiently

3. The skill to evaluate information and its source critically

4. The ability to incorporate selected information into one's knowledge base

5. The skill of using information effectively to accomplish a specific purpose

6. Understanding the economic, legal, and social issues surrounding the use of information and access and use information ethically and legally. ${ }^{40}$

AALL denotes five principles for research competency. They are:

1. Possess foundational knowledge of the legal system and legal information sources

2. Gathers information through effective and efficient research strategies

3. Critically evaluates information

4. Applies information effectively to resolve a specific issue or need

5. Distinguishes between ethical and unethical uses of information. ${ }^{41}$

Incorporate, as appropriate, these skills into your program. Use these skills and principles to assist you in developing goals and learning outcomes. Then assess the effectiveness of the program by engaging the audience to evaluate information using these skills.

\section{Consult the Experts-Existing Programs}

Avoid reinventing the wheel. Look for and discover programming that exists on this topic. Evaluate it for your intended purpose and audience, and then borrow what fits. A simple Google search reveals any number of LibGuides and information literacy programs. Are you seeking resources on the basics of information literacy or a more granular version of the topic such as media literacy? On the specific topic of media literacy and/or fake news consider the following by no means exhaustive list:

1. ALA Post Truth: Fake News and A New Era of Information Literacy ${ }^{42}$

2. Librarian Fights Fake News with Information Literacy ${ }^{43}$

${ }^{39} \mathrm{Id}$.

40 Association of College And Research Libraries, Standards Committee, Information Literacy Competency Standards for Higher Education, http://www.ala.org.

41 American Association of Law Libraries, Principles and Standards for Legal Research Competencies (2013).

${ }^{4}$ American Library Association, Post-Truth: Fake News and a New Era of Information Literacy (Jan. 6, 2017) http://www. ala.org/tools/programming/post-truth-fake-news-and-new-era-information-literacy.

${ }^{43}$ Ben Bugajski, Librarian Fights Fake News With Information Literacy (Dec. 11, 2017) https://www. Record.umich.edu. 
3. Information Literacy in an Era of Alternative Facts \& Fake News ${ }^{44}$

4. Information Literacy \& Fake News ${ }^{45}$

5. Fake News, Indiana University East ${ }^{46}$

6. News Sources, University of Michigan Library and ${ }^{47}$

7. Fake News, University of Virginia Library. ${ }^{48}$

Look for programming that addresses critical examination of source and content identification, finding information that fits the intended purpose in addition to the tried and true questions related to evaluation of any information for currency, expertise of the author, and accuracy. Examine programming for assessment of the skill. Consider how to update or tweak a portion of an existing program for the program you are designing. Features such as:

1. Upstream searching

2. News syndication and how content goes viral

3. Read laterally ${ }^{49}$

4. Use of journal impact factor as indicia of quality

5. Tools such as the Wayback Machine and the Internet Archive to track down original sources.

Look for programming that discusses what makes a news story false. Consider programming elements such as: inability to verify the claim made, stories that appeal to your emotions, lack of author expertise, and claims that cannot be replicated or supported independently or include inaccurate or false or non-existent citations. What skills does your audience require to "Fact Check Like a Pro"? Variations on this theme may be discussions on credentials, bias, and currency. Your goal is to educate a consumer to judge critically for fit, purpose, and veracity any piece of information they intend to use.

\section{E. Tools for the Consumer}

Give your audience the tools they need to be successful in critically examining information. Include in your presentation information on FactCheck.org, Snopes.com, Washington Post Fact Checker, Politifact, Hoax-Slayer. net, and NPR Fact-Check. This list is not intended to be exhaustive, nor does one have to present on each tool listed. Rather, this is an illustrative list of the types of tools that are available and that a consumer of news might use in ascertaining the quality of the work.

Each of these organizations/tools is unique but generally serves the goal of verifying the facts in the media discourse and as represented on the internet. Presenting several gives the user the ability to select the best tool based on the information. For example, Hoax-Slayer's focus is to debunk spam and internet hoaxes. Snopes began in 1994 with the intent of debunking urban legends. In contrast, Fact Check.org, Politifact, NPR Fact Check, and Washington Post Fact Check look at current news events. If the piece is international rather than domestic, different tools may be best suited to the inquiry. FACT Can and TrudeauMetre focus on Canadian issues, El Poligrafo, and The Hound address Mexico. Guardian Realty Check and BBC Reality Check are excellent tools for pieces generated in the United Kingdom. The focus, again, is selecting the appropriate tool for the specific query. Present a range of tools to enable the consumer to select the tool best suited to fact check their particular story. For example, CNN Caught Staging a Fake News Video ran rampant following the 2017 June London attacks. A simple internet search reveals that Snopes is on the case and disproves the claim that $\mathrm{CNN}$ is making rather than reporting the news. ${ }^{50}$

${ }^{44}$ Temple University Library, Information Literacy in an Era of Alternative Facts \& Fake News, https://www.guides. temple.edu.

${ }^{45}$ Kentucky Virtual Library, Information Literacy \& Fake News, https://www. KYVL.org

${ }^{46}$ Indiana University East, Fake News, http://www.iue.libguide.com.

${ }^{47}$ University of Michigan Library, News Sources, https://www.University of Michigan Library at http://guides.lib.umich. edu/c.php?g=283063\&p=447174.

${ }^{48}$ University of Virginia Library, Fake News, https:/guides.lib.virginia.edu/c.php?g=600315\&p=4156721.

${ }^{49}$ When using the web to locate information, reading laterally is the practice of investigating what other web sites say about a page as an indicia of authority and reliability. What "Reading Laterally" Means, https://webliteracy.pressbooks.com/chapter/ what-reading-laterally-means/ (last visited March 21, 2018 at 7:59 AM).

${ }^{50}$ CNN Staged Muslim Protest Attack? Pundits and Conspiracy Theorists Claimed that CNN Staged a Demonstration in London Involving Muslims After a June 2017 Attack, https://Snopes.com (last visited March 21, 2018). 


\section{Conclusion}

The need for information- and media-literate consumers is significant in today's web-focused environment with a 24-hour news cycle. The most recent United States presidential election highlighted the fabrication of news and made fake news a buzzword. Existing efforts at educating persons to be cautious consumers are insufficient down to a lack of attention, an absence of awareness, a desire to trust what one likes, and an increasingly sophisticated business model surrounding the fabrication of news. The need for effective education is real. The need to build sophisticated consumers of news is critical.

Effective information literacy programming with a focus on fake news draws much from existing programming, but must include specific elements unique to news that highlights the nuances of the news industry. We can borrow much from our colleagues in the journalism profession as to what makes a quality news story. Reinforcing the traditional programmatic themes of source evaluation, currency, evaluation of expertise, teaching consumers to engage in a critical examination of the content and discussion of available tools for verifying content, combined with an understanding of how one consumes news, creates an effective program for educating media literate consumers. 